JETP Letters

\title{
Manifestation of geometric effects in temperature behavior of AC magnetic response of Josephson Junction Arrays
}

\author{
S. Sergeenkov ${ }^{1,2}$ and F.M. Araujo-Moreira ${ }^{1}$ \\ 1 Departamento de Física e Engenharia Física, Grupo de Materiais e Dispositivos, \\ Centro Multidisciplinar para o Desenvolvimento de Materiais Cerâmicos, \\ Universidade Federal de $S$ ao Carlos, S ao Carlos, SP, 13565-905 Brazil \\ 2 Bogoliubov Laboratory of Theoretical Physics, Joint Institute for Nuclear Research, \\ Dubna 141980, Moscow Region, Russia
}

\begin{abstract}
By improving resolution of home-made mutual-inductance measurements technique, a pronounced step-like structure (with the number of steps $n=4$ for all AC fields) has been observed in the temperature dependence of AC susceptibility in artificially prepared two-dimensional Josephson Junction Arrays (2D-JJA) of unshunted $\mathrm{Nb}-\mathrm{AlO}_{x}-\mathrm{Nb}$ junctions with $\beta_{L}(4.2 \mathrm{~K})=30$. Using a single-plaquette approximation of the overdamped 2D-JJA model, we were able to successfully fit our data assuming that steps are related to the geometric properties of the plaquette. The number of steps $n$ corresponds to the number of flux quanta that can be screened by the maximum critical current of the junctions. The steps are predicted to manifest themselves in arrays with the inductance related parameter $\beta_{L}(T)$ matching a "quantization" condition $\beta_{L}(0)=2 \pi(n+1)$.
\end{abstract}

PACS: 74.25.Ha, 74.50.+r, 74.80.Bj

1. Introduction. Many unusual and still not completely understood magnetic properties of Josephson Junction Arrays (JJAs) continue to attract attention of both theoreticians and experimentalists alike (for recent reviews on the subject see, e.g. 1, 2, 3, 4, and further references therein). In particular, among the numerous spectacular phenomena recently discussed and observed in JJAs we would like to mention the dynamic temperature reentrance of AC susceptibility [2] (closely related to paramagnetic Meissner effect [3]) and avalanche-like magnetic field behavior of magnetization [4, 5] (closely related to self-organized criticality (SOC) [6, 7]). More specifically, using highly sensitive SQUID magnetometer, magnetic field jumps in the magnetization curves associated with the entry and exit of avalanches of tens and hundreds of fluxons were clearly seen in SIS-type arrays [5]. Besides, it was shown that the probability distribution of these processes is in good agreement with the SOC theory [7. An avalanche character of flux motion was observed at temperatures at which the size of the fluxons did not exceed the size of the cell, that is, for discrete vortices. On the other hand, using a similar technique, magnetic flux avalanches were not observed in SNS-type proximity arrays [ 8 ] despite a sufficiently high value of the inductance $L$ related critical parameter $\beta_{L}=2 \pi L I_{C} / \Phi_{0}$ needed to satisfy the observability conditions of SOC. Instead, the observed quasi-hydrodynamic flux motion in the array was explained by the considerable viscosity characterizing the vortex motion through the Josephson junctions.
In this paper we present experimental evidence for manifestation of novel geometric effects in magnetic response of high-quality ordered 2D-JJA. By increasing the resolution of our home-made mutual-inductance measurements technique, we were able to observe for the first time a fine, step-like structure (with the number of steps $n=4$ for all AC fields used in our experiments) in the temperature behavior of AC susceptibility in artificially prepared 2D-JJA of unshunted $\mathrm{Nb}-\mathrm{AlO}_{x}-\mathrm{Nb}$ junctions. Using a single-plaquette approximation of the overdamped 2D-JJA model, we show that the number of steps $n$ corresponds to the number of flux quanta that can be screened by the maximum critical current of the junctions and as a result steps will manifest themselves in arrays with the inductance related parameter $\beta_{L}(T)$ matching a "quantization" condition $\beta_{L}(0)=2 \pi(n+1)$.

2. Experimental results. To measure the complex AC susceptibility in our arrays with high precision, we used a home-made susceptometer based on the so-called screening method in the reflection configuration 9 10, 11. The experimental system was calibrated by using a high-quality niobium thin film. Previously, we have shown that the calibrated output of the complex voltage in this experimental setup corresponds to the true complex AC susceptibility (for more details on the experimental technique and setups used in our study, see [2, 11]).

Measurements were performed as a function of the temperature $T$ (for $1.5 K<T<15 K$ ), and the am- 
plitude of the excitation field $h_{a c}$ (for $1 m O e<h_{a c}<$ $10 O e$ ) normal to the plane of the array. The frequency of AC field in the experiments reported here was fixed at $20 \mathrm{kHz}$. The used in the present study unshunted 2D-JJAs are formed by loops of niobium islands (with $\left.T_{C}=9.25 \mathrm{~K}\right)$ linked through $\mathrm{Nb}-\mathrm{AlO}_{x}-\mathrm{Nb}$ Josephson junctions and consist of $100 \times 150$ tunnel junctions. The unit cell has square geometry with lattice spacing $a=46 \mu \mathrm{m}$ and a single junction area of $5 \times 5 \mu \mathrm{m}^{2}$. Since the inductance of each loop is $L=\mu_{0} a=64 \mathrm{pH}$ and the critical current of each junction is $I_{C}(4.2 K)=$ $150 \mu A$, we have $\beta_{L}(4.2 K)=30$. Recall that parameter $\beta_{L}(T)=2 \pi L I_{C}(T) / \Phi_{0}$ (where $\Phi_{0}$ is the magnetic flux quantum) is proportional to the number of flux quanta that can be screened by the maximum critical current in the junctions.

It is important to mention that magnetic field dependence of the critical current of the array (taken at $T=$ $4.2 \mathrm{~K}$ ) on DC magnetic field $H_{d c}$ (parallel to the plane of the sample) exhibited [2, 11] a sharp Fraunhofer-like pattern characteristic of a single-junction response, thus proving a rather strong coherence within arrays (with negligible distribution of critical currents and sizes of the individual junctions) and hence the high quality of our samples.

The observed temperature dependence of the real part of AC susceptibility for different AC fields is shown in Fig. 1 A pronounced step-like structure is clearly seen at higher temperatures. The number of steps $n$ does not depend on AC field amplitude and is equal to $n=4$. As expected [2] 11, 12, for $h_{a c}>40 m O e$ (when the array is in the mixed-like state with practically homogeneous flux distribution) the steps are accompanied by the previously observed reentrant behavior with $\chi^{\prime}\left(T, h_{a c}\right)$ starting to increase at low temperatures.

3. Discussion. To understand the step-like behavior of the AC susceptibility observed in unshunted 2D-JJAs, in principle one would need to analyze in detail the flux dynamics in these arrays. However, as we have previously reported 2, 11, 12, because of the welldefined periodic structure of our arrays with no visible distribution of junction sizes and critical currents, it is quite reasonable to assume that the experimental results obtained from the magnetic properties of our 2D-JJAs could be understood by analyzing the dynamics of just a single unit cell (plaquette) of the array. As we shall see, theoretical interpretation of the presented here experimental results based on single-loop approximation, is in excellent agreement with the observed behavior. In our analytical calculations, the unit cell is a loop containing four identical Josephson junctions and the

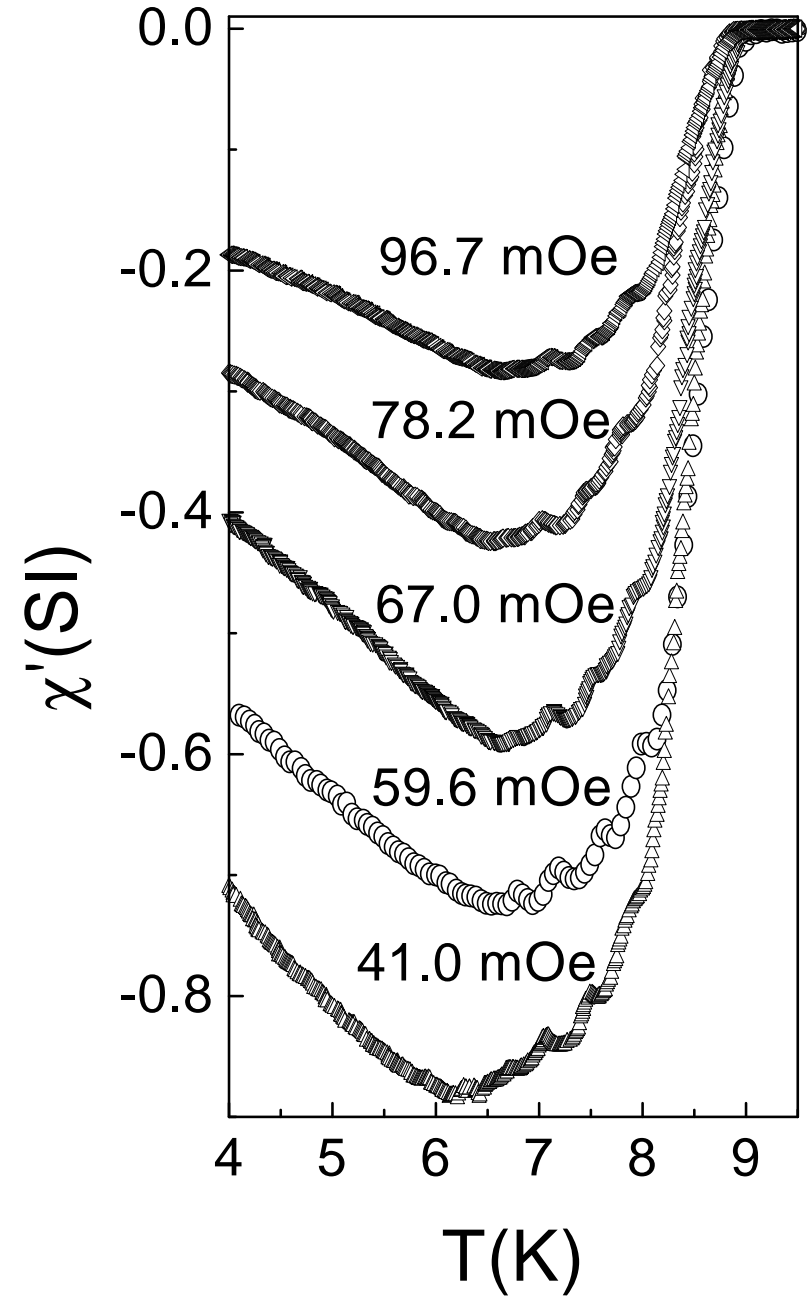

Fig 1 Experimental results for temperature dependence of the real part of AC susceptibility $\chi^{\prime}\left(T, h_{a c}\right)$ for different $\mathrm{AC}$ field amplitudes $h_{a c}=41.0,59.6,67.0$, 78.2 and $96.7 m O e$.

measurements correspond to the zero-field cooling AC magnetic susceptibility. If we apply an AC external field $H_{a c}(t)=h_{a c} \cos \omega t$ normally to the 2D-JJA, then the total magnetic flux $\Phi(t)$ threading the four-junction superconducting loop is given by $\Phi(t)=\Phi_{\text {ext }}(t)+L I(t)$ where $L$ is the loop inductance, $\Phi_{\text {ext }}(t)=S H_{a c}(t)$ is the flux related to the applied magnetic field (with $S \simeq a^{2}$ being the projected area of the loop), and the circulating current in the loop reads $I(t)=I_{C}(T) \sin \phi(t)$. Here $\phi(t)$ is the gauge-invariant superconducting phase difference across the $i$ th junction. As is well-known, in the case of four junctions, the flux quantization condition reads [1, 13]

$$
\phi=\frac{\pi}{2}\left(n+\frac{\Phi}{\Phi_{0}}\right)
$$




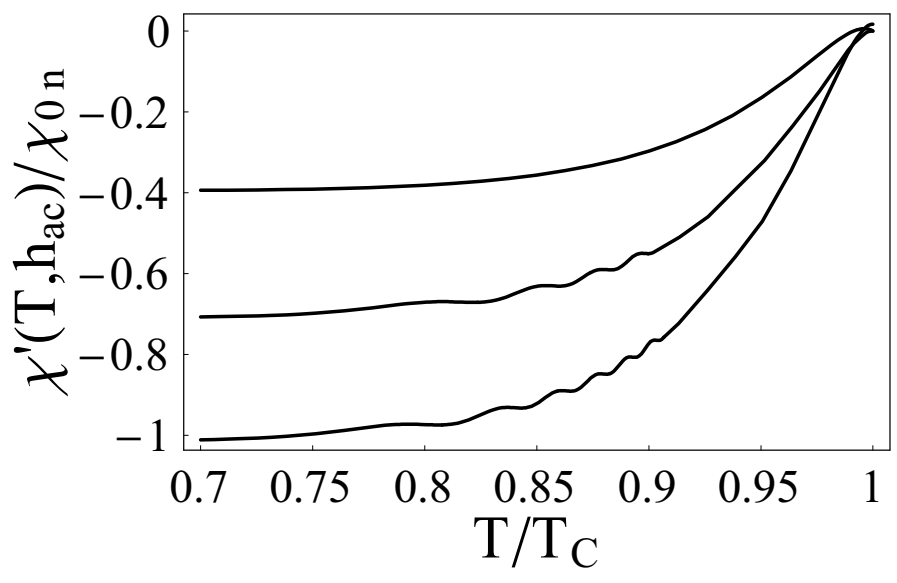

Fig[2] Theoretically predicted dependence of the normalized susceptibility $\chi^{\prime}\left(T, h_{a c}\right) / \chi_{0 n}$ (for better visual effect the curves are normalized differently with $\chi_{00}=$ $2.5 \chi_{0}, \chi_{03}=1.5 \chi_{0}$, and $\chi_{05}=\chi_{0}$ where $\left.\chi_{0}=S^{2} / V L\right)$ on reduced temperature $T / T_{C}$ according to Eqs.(3)-(5) for $f=0.5$ and for "quantized" values of $\beta_{L}(0)=$ $2 \pi(n+1)$ (from top to bottom): $n=0,3$ and 5 .

where $n$ is an integer and for simplicity we assume as usual that [2] 11] $\phi_{1}=\phi_{2}=\phi_{3}=\phi_{4} \equiv \phi$.

To properly treat the magnetic properties of the system, let us introduce the following Hamiltonian

$$
\mathcal{H}(t)=J(T)[1-\cos \phi(t)]+\frac{1}{2} L I^{2}(t)
$$

which describes the tunneling (first term) and inductive (second term) contributions to the total energy of a single plaquette. Here, $J(T)=\left(\Phi_{0} / 2 \pi\right) I_{C}(T)$ is the Josephson coupling energy.

Before turning to the interpretation of the observed step-like structure of $\chi^{\prime}\left(T, h_{a c}\right)$, we would like to briefly comment on the origin of reentrant behavior in our unshunted arrays (which has been discussed in much detail earlier, see 2, 12]). A comparative study of the magnetic properties of two-dimensional arrays of both unshunted and shunted (using a molybdenum shunt resistor short-circuiting each junction) $\mathrm{Nb}-\mathrm{AlO}_{x}-\mathrm{Nb}$ junctions revealed [12] that the dynamic reentrance phenomenon can be explained by properly addressing the (neglected in our overdamped model) damping effects associated with the finite value of the StewartMcCumber parameter $\beta_{C}(T)=2 \pi C_{J} R_{J}^{2} I_{C}(T) / \Phi_{0}$ (where $C_{J}$ is the capacitance and $R_{J}$ is the quasiparticle resistance of the array). More precisely, the reentrance was found to take place in considered here unshunted arrays (with $\left.\beta_{C}(4.2 K)=30\right)$ and totally disappeared in shunted arrays (with $\beta_{C}(4.2 K)=1$ ). It is important to mention that both arrays had the same value of the $\beta_{L}$ parameter, namely $\beta_{L}(4.2 K)=30$.

Returning to the discussion of the observed here geometrical effects, we notice that the number of observed steps $n$ (in our case $n=4$ ) clearly hints at a possible connection between the observed here phenomenon and flux quantization condition within a single fourjunction plaquette. Indeed, the circulating in the loop current $I(t)=I_{C}(T) \sin \phi(t)$ passes through its maximum value whenever $\phi(t)$ reaches the value of $\frac{\pi}{2}(2 n+1)$ with $n=0,1,2 \ldots$ As a result, the maximum number of fluxons threading a single plaquette (see Eq.(1)) over the period $2 \pi / \omega$ becomes equal to $\langle\Phi(t)\rangle=(n+1) \Phi_{0}$. In turn, the latter equation is equivalent to the following condition $\beta_{L}(T)=2 \pi(n+1)$. Since this formula is valid for any temperature, we can rewrite it as a geometrical "quantization" condition $\beta_{L}(0)=2 \pi(n+1)$. Recall that in our array $\beta_{L}(0)=31.6$ (extrapolated from its experimental value $\left.\beta_{L}(4.2 K)=30\right)$ which is a perfect match for the above "quantization" condition predicting $n=4$ for the number of steps in a single plaquette, in excellent agreement with the observations.

Based on the above discussion, we conclude that in order to reproduce the observed temperature steps in the behavior of AC susceptibility, we need a particular solution to Eq.(1) for the phase difference in the form of $\phi_{n}(t)=\frac{\pi}{2}(2 n+1)+\delta \phi(t)$ assuming $\delta \phi(t) \ll 1$. After substituting this Ansatz into Eq.(1), we find that $\phi_{n}(t) \simeq \frac{\pi}{2} n+\frac{1}{4} \beta_{L}(T)+\frac{1}{4} f \cos \omega t$ where $f=2 \pi S h_{a c} / \Phi_{0}$ is the AC field related frustration parameter. Using this effective phase difference, we can calculate the $\mathrm{AC}$ response of a single plaquette. Namely, the real part of susceptibility reads

$$
\chi^{\prime}\left(T, h_{a c}\right)=\frac{1}{\pi} \int_{0}^{\pi} d(\omega t) \cos (\omega t) \chi_{n}(t)
$$

where

$$
\chi_{n}(t)=-\frac{1}{V}\left[\frac{\partial^{2} \mathcal{H}}{\partial h_{a c}^{2}}\right]_{\phi=\phi_{n}(t)}
$$

Here $V$ is the sample's volume.

For the explicit temperature dependence of $\beta_{L}(T)=$ $2 \pi L I_{C}(T) / \Phi_{0}$ we use the well-known [14, 15] analytical approximation of the BCS gap parameter (valid for all temperatures), $\Delta(T)=\Delta(0) \tanh \left(2.2 \sqrt{\frac{T_{C}-T}{T}}\right)$ with $\Delta(0)=1.76 k_{B} T_{C}$ which governs the temperature dependence of the Josephson critical current

$$
I_{C}(T)=I_{C}(0)\left[\frac{\Delta(T)}{\Delta(0)}\right] \tanh \left[\frac{\Delta(T)}{2 k_{B} T}\right]
$$

Fig. 2 depicts the predicted by Eqs.(3)-(5) dependence of the AC susceptibility on reduced temperature for 


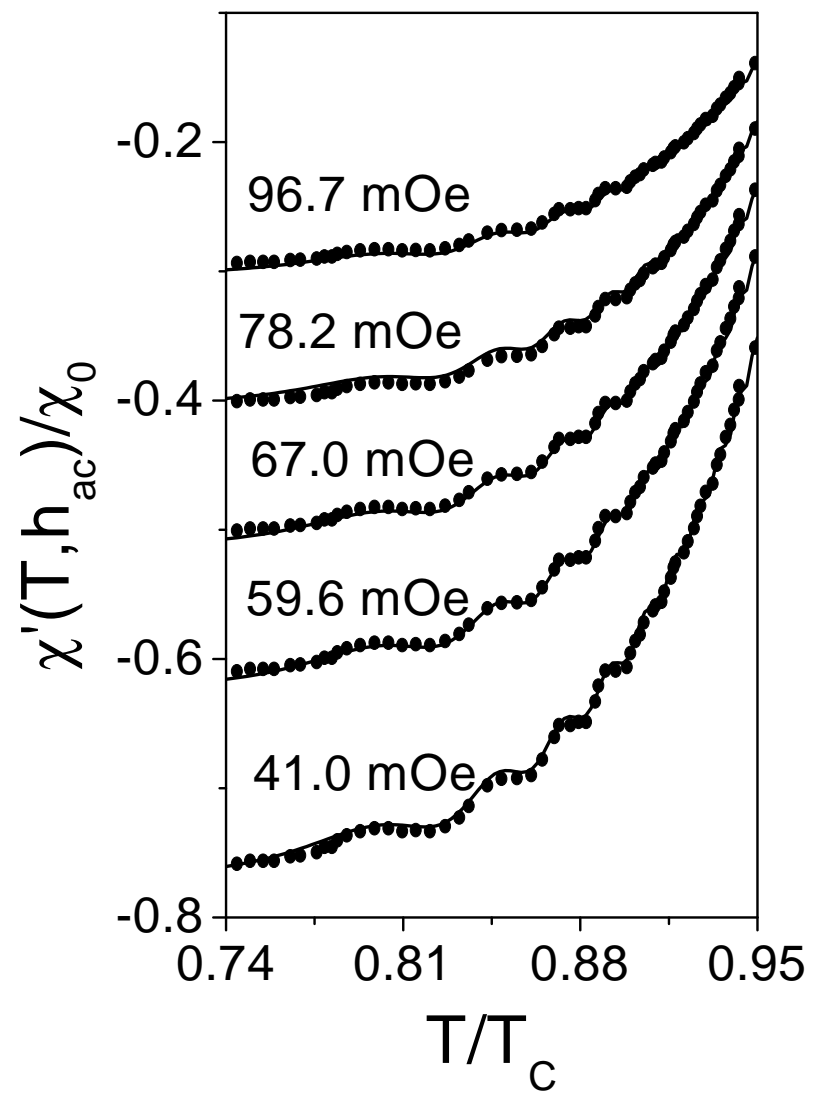

Fig[3 Fits (solid lines) of the experimental data for $h_{a c}=41.0,59.6,67.0,78.2$, and $96.7 m O e$ according to Eqs.(3)-(5) with $\beta_{L}(0)=10 \pi$.

$f=0.5$ and for different "quantized" values of $\beta_{L}(0)=$ $2 \pi(n+1)$. Notice the appearance of three and five steps for $n=3$ and $n=5$, respectively (as expected, case $n=0$ corresponds to a smooth temperature behavior without steps).

In Fig. 3 we present fits (shown by solid lines) of the observed temperature dependence of the normalized susceptibility $\chi^{\prime}\left(T, h_{a c}\right) / \chi_{0}$ for different magnetic fields $h_{a c}$ according to Eqs.(3)-(5) using $\beta_{L}(0)=$ $10 \pi$. As is seen, our simplified model based on a single-plaquette approximation demonstrates an excellent agreement with the observations.

In summary, a step-like structure (accompanied by previously seen low-temperature reentrance phenomenon) has been observed for the first time in the temperature dependence of AC susceptibility in artificially prepared two-dimensional Josephson Junction Arrays of unshunted $\mathrm{Nb}-\mathrm{AlO} \mathrm{O}_{x}-\mathrm{Nb}$ junctions. The steps are shown to occur in arrays with the inductance related parameter $\beta_{L}(T)$ matching the "quantization" condition $\beta_{L}(0)=2 \pi(n+1)$ where $n$ is the number of steps.
We thank W. Maluf for his help in running the experiments. We are indebted to P. Barbara, C.J. Lobb, R.S. Newrock, and A. Sanchez for useful discussions. The authors gratefully acknowledge financial support from Brazilian Agency FAPESP under grant 2003/00296-5.

1. R.S. Newrock, C.J. Lobb, U. Geigenmuller, and M. Octavio, Solid State Physics 54, 263 (2000); Mesoscopic and Strongly Correlated Electron Systems-II, Ed. by M.V. Feigel'man, V.V. Ryazanov and V.B. Timofeev, Phys. Usp. (Suppl.) 44 (10) (2001); S. Sergeenkov, in Studies of High temperature Superconductors, Ed. by A.V. Narlikar (Nova Science Publishers, New York, 2001), vol. 39, p. 117.

2. F.M. Araujo-Moreira, P. Barbara, A.B. Cawthorne, and C.J. Lobb, in Studies of High temperature Superconductors, Ed. by A.V. Narlikar (Nova Science Publishers, New York, 2002), vol. 43, p. 227.

3. M.S. Li, Phys. Rep. 376, 133 (2003).

4. E. Altshuler and T.H. Johansen, Rev. Mod. Phys. 76, 471 (2004).

5. S.M. Ishikaev, E.V. Matizen, V.V. Ryazanov, V.A. Oboznov, and A.V. Veretennikov, JETP Lett. 72, 26 (2000).

6. H.J. Jensen, Self Organized Criticality: Emergent Complex Behavior in Physical and Biological Systems (Cambridge University Press, Cambridge, 1998).

7. S.L. Ginzburg and N.E. Savitskaya, JETP Lett. 73, 145 (2001).

8. S.M. Ishikaev, E.V. Matizen, V.V. Ryazanov, and V.A. Oboznov, JETP Lett. 76, 160 (2002).

9. B. Jeanneret, J.L. Gavilano, G.A. Racine, Ch. Leemann, and P. Martinoli, Appl. Phys. Lett. 55, 2336 (1989).

10. P. Martinoli and C. Leeman, J. Low Temp. Phys. 118, 699 (2000).

11. F.M. Araujo-Moreira, P. Barbara, A.B. Cawthorne, and C.J. Lobb, Phys. Rev. Lett. 78, 4625 (1997); P. Barbara, F.M. Araujo-Moreira, A.B. Cawthorne, and C.J. Lobb, Phys. Rev. B 60, 7489 (1999).

12. F.M. Araujo-Moreira, W. Maluf, and S. Sergeenkov, unpublished.

13. A. Barone and G. Paterno, Physics and Applications of the Josephson Effect (A Wiley-Interscience Publisher, New York, 1982).

14. R. Meservey and B.B. Schwartz, in Superconductivity, vol.1, ed. by R.D. Parks (M. Dekker, New York, 1969), p.117.

15. S. Sergeenkov, JETP Lett. 76, 170 (2002). 